\title{
Development of an Effective Public Health Screening Program to Assess Hearing Disabilities among Newborns in Shanghai: A Prospective Cohort Study
}

Xiaoming Sun, MD PhD, Shanghai Municipal Health Bureau, Shanghai, China; School of Public Health, Harvard University, Boston, MA,USA

Xiaoming Shen, MD PhD, Xinhua Hospital, Jiaotong University, Shanghai, China

David Zakus, PhD, Dalla Lana School of Public Health and Department of Health Policy, Management and Evaluation, Faculty of Medicine, University of Toronto, Canada Jun Lv, PhD, School of Public Health, Fudan University, Shanghai, China

Zhengmin Xu, MD PhD, Pediatric Hospital, Fudan University. Shanghai, China Hao Wu, MD PhD, Xinhua Hospital, Jiaotong University, Shanghai, China William Hsiao, PhD, School of Public Health, Harvard University, Boston, MA, USA

Xiaoming Sun, Shanghai Municipal Health Bureau, 1477 Beijing West Road, Shanghai, China 200040, Email: xm_sun2003@yahoo.com.cn

\footnotetext{
Abstract

\section{Background}

An effective, systematic program of screening, diagnosis and intervention against hearing loss in infants could help them avoid developmental impediments and could help society stem preventable healthcare burdens. We assessed the feasibility and outcomes of a new public health universal newborn hearing screening program (UNHSP) for neonates born in Shanghai.
}

\section{Methods}

From March 2002 to June 2007, we conducted a two-stage hearing loss screening program for neonates born at all 105 delivery hospitals in Shanghai. Institutional participants in the program 
followed standardized testing criteria and procedures. The first stage of screening occurred in the hospital during the third day post-birth; positive infants underwent a second-stage outpatient screening on Day 42. Positive infants were examined at clinical diagnosis centres, and interventions were conducted at rehabilitation centres. In 2003, a random sample of parents were interviewed about the program, and their level of stress over learning their child screened positive were recorded and analyzed.

\section{Findings}

Overall, our program screened $72.98 \%$ of eligible infants and provided effective interventions within six months to $86.31 \%$ of those with hearing loss. During the 5 -year study, first-stage screenings assessed $90.85 \%$ of 616,880 eligible infants and found $12.16 \%$ positive. Day 42 screenings had a $65.68 \%$ participation rate and a positive rate of $14.75 \%$. Of these, $0.146 \%$ were deemed permanently hearing impaired within three months of birth. Parental satisfaction measures exceeded $90 \%$.

\section{Interpretation}

A government-sponsored public health program to screen, diagnose, treat and provide interventions for all newborns with permanently hearing impairment can be effectively implemented and can achieve outcomes that surpass comparable clinical initiatives.

\section{Introduction}

Worldwide, significant bilateral hearing loss is found in one to three of every 1000 newborns in wellbaby populations and in $2 \%$ to $4 \%$ of infants in neonatal intensive care units (American Academy of Pediatrics 1999; Green et al. 1999; Zhao 2001). Failure to detect hearing loss in a timely manner, and to intervene as soon as possible, can lead to activity limitations in linguistic and language functioning and cognitive development, and to difficulties in social participation. Research indicates that diagnosing hearing loss in newborns within three months of birth and implementing appropriate treatment and interventions within six months of birth normalizes cognitive and language development, thereby allowing hearing-impaired infants to develop at a pace similar to that of infants without hearing loss. Such studies also show that the long-term quality of life for infants who receive interventions surpasses what would have been possible had treatment been delayed or absent (American Academy of Pediatrics and Joint Committee on Infant Hearing 1995; Shen 2001).

Research also shows more traditional detection efforts are inadequate: only about $50 \%$ of neonates with congenital hearing loss are detected using the high-risk registry that screens for, among other items, a family history of deafness. In addition, physician examinations and parental observations only occasionally succeed in identifying congenital hearing loss in infants under the age of one. To improve the early detection of infant hearing loss, in the past 15 years many countries and cities have instituted universal newborn hearing screening programs (UNHSPs) or have set up a series of screening measures (American Academy of Pediatrics 1999; American Academy of Pediatrics and Joint Committee on Infant Hearing 1995; Kanne et al. 1999; Wessex Universal Neonatal Hearing Screening Trial Group 1998; Grandori 1999).

In the United States and elsewhere (White and Behrens 1993), UNHSPs have spread gradually (White 2003; Morton and Nance 2006; Wu 2000), spurred by the endorsements of the 1993 National Institutes of Health Consensus Development Conference and, in 1994, by the American Academy of Pediatrics (AAP). The AAP's findings set a goal of universal detection of hearing loss in infants before 3 months of age, coupled with appropriate intervention no later than 6 months of age. Both groups advocated hearing loss screening for all newborns before hospital discharge (American Academy of Pediatrics 1999; American Academy of Pediatrics and Joint Committee on Infant Hearing 1995; National Institutes of Health 1993; Centers for Disease Control and Prevention 2003).

In 2003, an analysis of UNHSPs in the United States showed 58\% of hospitals employed a two-stage process: initial inpatient screening after birth in the hospital, followed by outpatient 
screening before three months of age. This achieved a first-stage screening rate of $70 \%$ among eligible newborns. After the second screening, $56 \%$ of the infants were referred for diagnosis (by three months of age), and $53 \%$ of the infants diagnosed with hearing loss had received an appropriate intervention by six months of age (White 2003).

Most clinical UNHSPs in developed countries use otoacoustic emission (OAE) screening protocols, which include some combination of transient-evoked otoacoustic emission (TEOAE), distortion products otoacoustic emission (DPOAE) and automated auditory brainstem response (AABR) (Xu et al. 2003; Li et al. 2004; Shen 2002). Approximately 53\% of US screening programs use TEOAE and 67\% use AABR, with some employing both methods (White 2003). In general, these programs start with an initial screening test 24 to 48 hours following birth and either undertake a second inpatient or outpatient screening for newborns who test positive in the first stage or refer newborns with positive results directly to a diagnosis centre (US Preventive Services Task Force 2001 and 2002; Uus and Bamford 2006; Lin et al. 2002).

According to an epidemiological survey undertaken in Shanghai, between 600 and 700 of the 78,762 infants born in 1999 had some congenital impairment. Furthermore, the rate of disability for children under seven years of age was $0.968 \%$, with $16 \%$ of that group experiencing hearing loss (Zhao et al. 1999).

In 2000, Shanghai's government health bureau initiated a strategy for developing effective monitoring and intervention techniques that might limit or prevent disabilities in newborns. The strategy concentrates on three areas of public health importance: genetic testing and counselling before pregnancy, prenatal screening for congenital impairments (such as Down's syndrome) and newborn screening for hearing loss. To address the strategy's hearing loss screening component, we developed a public health universal hearing loss screening procedure that was systematically designed to ensure cross-program consistency in all key aspects: instrumentation, diagnosis and screening procedures, interventions, administration and organization, personnel training and quality control of methods and outcomes. Overall, we sought to ensure our program would meet the needs of Shanghai's population and meet, or even exceed, world standards.

\section{Methods}

\section{Network Infrastructure}

All 105 of Shanghai's delivery hospitals, both obstetrics and gynecology hospitals and obstetrics and gynecology departments in general hospitals, participated in the study. Diagnostic centres were set up in three institutions: Shanghai Children's Medical Centre and Xinhua Hospital, both affiliated with Jiaotong University; and Fudan Pediatric Hospital, affiliated with Fudan University. Rehabilitation centres were set up at Xinhua Hospital, the EENT hospital affiliated with Fudan University, and Shanghai Municipal Federation of Disabled People, a rehabilitation centre that assists those with disabilities. The 19 district-level women and children healthcare networks assisted in managing this stage of the process. Administration was centralized at the Shanghai Institute for Women and Children Healthcare, an institute organized and supported by the Shanghai Municipal Health Bureau.

In order to standardize screening results, in 2001 we purchased 130 units of the GSI 60 TEOAE system (Grasen-Stadler GSI 60, Grasen-Stadler, Inc., A Welch Allyn Co., Milford, NJ, USA). This instrument was selected after an analysis of its technical performance and cost, a review that used reports on its ease of use and clinical performance from experts in hearing screening systems. We adopted the World Health Organization's criteria for hearing screening (see Table 2). Regular training sessions were arranged for the hospital personnel who would be conducting the screenings, and participants were granted work permits (or licenses) upon passing a proficiency examination. Nominal testing fees of $20 \mathrm{RMB}$ (US 2.50 dollars) for one ear and 40 RMB (US 5.00 dollars) for both ears were set. Standardized processes were established for information collection and quality control.

\section{Pilot Study}

We carried out a 1-year pilot study (September 2000 to August 2001) to determine participation 
rates for screenings on an outpatient basis alone (Day 42) and for inpatient screenings conducted on either the first, second or third day following birth. In addition, we conducted an internal comparison of the inpatient results, including images of the outer surfaces of the infants' tympanums and results from tests using high-frequency acoustic impedance technology, to determine the status of eardrum effusion in neonates assessed on each of the three days. A total of 5000 infants were screened in the pilot study: 2000 during outpatient checkups at birth hospitals on Day 42 and 1000 on each of the first, second and third days following birth prior to hospital discharge.

Timing for inpatient screenings was chosen according to the hospitalization period after spontaneous delivery, which is usually three days in Shanghai. Follow-up screening took place on the 42nd day following birth, the fixed time for the first return checkup of newborns and their mothers in the birth hospital. The days of hospitalization duration and return checkup are settled by regulations of Shanghai Municipality on Mother and Infant Health Care. The timing was very critical to effectively conduct the program.

Based on the results of the pilot studies we produced two documents: The Strategy of Launching Universal Newborn Hearing Screening Program in Shanghai and The Plan for Universal Newborn Hearing Screening, Diagnosis and Intervention in Shanghai. These documents detailed the objectives, methods and requirements necessary for an effective screening program, the screening procedure (Figure 1) and the diagnosis and intervention processes (Figure 2) that such a program should follow, as well as the quality controls, administrative responsibilities and costs to both families and program. The documents and their recommendations were adopted by the Shanghai Municipal Health Bureau in early 2002 and used as the blueprint for implementing our city-wide UNHSP feasibility study that would address the public health needs of Shanghai infants who are born with hearing loss. To emphasize its powerful support for our large feasibility study of the hearing loss screening program, the Shanghai Municipal Health Bureau officially launched the program on March 3, 2002, in conjunction with World Hearing Day.

\section{Screening Feasibility Study}

From March 2002 through June 2007, 560,432 infants born in any of the study's 105 delivery hospitals were screened for hearing loss. Infants receiving positive reports received a second screening on Day 42 following birth, conducted on an outpatient basis at the infant's birth hospital. All screenings were performed by hospital personnel trained in the use of the equipment. Infants receiving a second positive report were referred to one of the three diagnostic centres in the study, where they were given an otoscopic exam, checked for the presence of tympanic effusion, and given DPOAE, $\mathrm{AABR}$ and hearing behaviour assessments (Figure 2).

Interventions included the use of hearing aids within six months of diagnosis for infants with slight to moderate hearing loss as well as for some with severe hearing loss. Interventions for infants with severe and extremely severe hearing loss consisted of the surgical implantation of artificial cochlear devices within 1 year of birth. Implantations were performed at two hospitals, and all infants who needed implants received them. In addition, all infants who received interventions had aural and language training at one of the three rehabilitation centres participating in the study.

\section{Sociological Measures}

In 2003, at six of the participating hospitals, 400 parents, either the father or mother of infants who had received positive results in first-stage screening, were randomly selected for face-to-face interviews. Interview questions were designed to assess parental satisfaction with the screening process. In addition, 110 of the 400 parents responded to questions that would help assess the level of anxiety or stress they felt upon learning their infants had initially screened positive for hearing loss.

\section{Results}

A comparison of data from the pilot study indicated first-stage inpatient screenings would be most effective, that is, less likely to result in false-positive reports, if conducted on the third day following 
birth. Imaging of the outer surface of the tympanum and acoustic checks using high-frequency impedance detection technology showed tympanic effusion rates decreased dramatically in the 3-day period following birth. According to our data, an effusion rate of up to $70 \%$ was found in the 1000 infants screened on the first day post-birth. This rate dropped to $30 \%$ in infants screened the second day and approached zero in the 1000 infants screened on the third day post-birth. Since the presence of amniotic fluid interfered with acoustic transmissions, we determined that TEOAE screenings would yield the fewest false-positive results if conducted on the third day following birth.

Figure 1. Administrative process for universal newborn hearing screening program in Shanghai

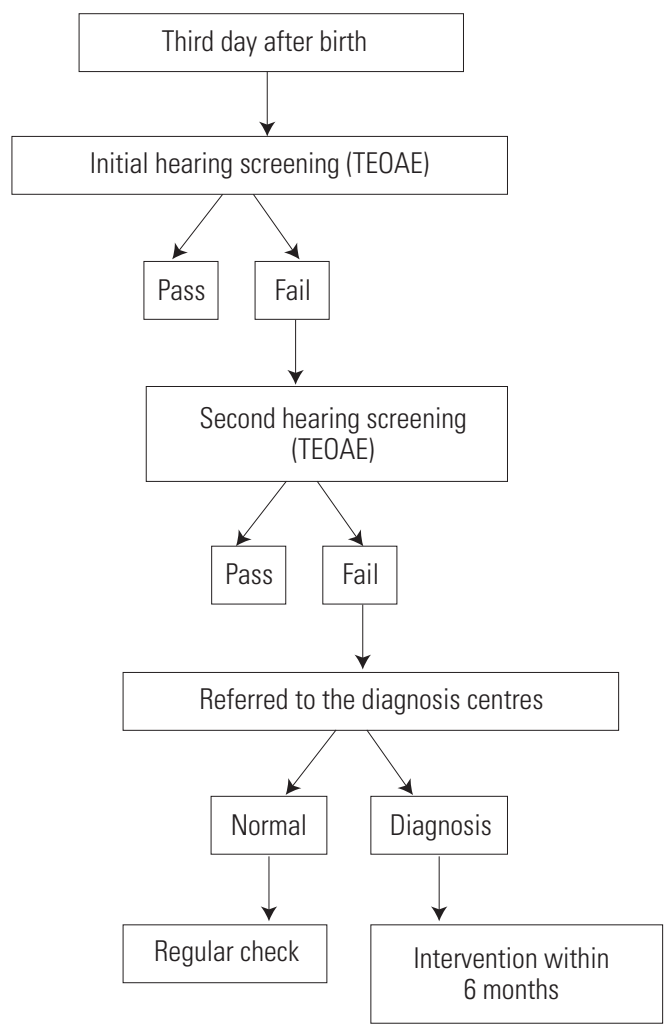

Our preliminary assessment also supported the two-stage screening process begun on the third day following birth and repeated if needed on Day 42. We found that initial inpatient screening achieved high participation but also produced a high number of false positives. Families receiving a positive diagnosis were motivated to attend the second screening; the majority of Chinese families have one child, so learning that the child may be hearing impaired caused parents great anxiety. The second screening, with its promise of further defining, perhaps even nullifying, the initial diagnosis, spurred compliance. In addition, by ensuring diagnosis by three months of age and intervention by six months, families were encouraged to pursue the timely potential benefits of participation in the program.

By lessening the number of false positives, the two-stage screening also greatly reduced the number of infants referred for diagnosis. If the $13.14 \%$ of infants who screened positive in the initial stage had all been referred for diagnosis, we estimate we would have needed 12 diagnosis centres to handle the referral load. With the two-stage testing, referrals totalled fewer than 2000 per year, a number that could be handled by three centres efficiently and well.

During the study period, 616,880 infants were born in delivery hospitals in Shanghai. Of that 
group of eligible infants, $90.85 \%(560,432)$ received an initial hearing screening test on the third day after birth, with $12.16 \%(68,152)$ testing positive for hearing loss. A second-stage screening, conducted on an outpatient basis 42 days following birth, had a participation rate of $65.68 \%(44,763$ infants). Positive results were reported in 14.75\% (6601) of the infants. Overall, the two-stage screening process achieved a $72.98 \%$ screening rate among eligible infants (Figure 3, Table 1).

Figure 2. Process for diagnosing hearing loss in newborns

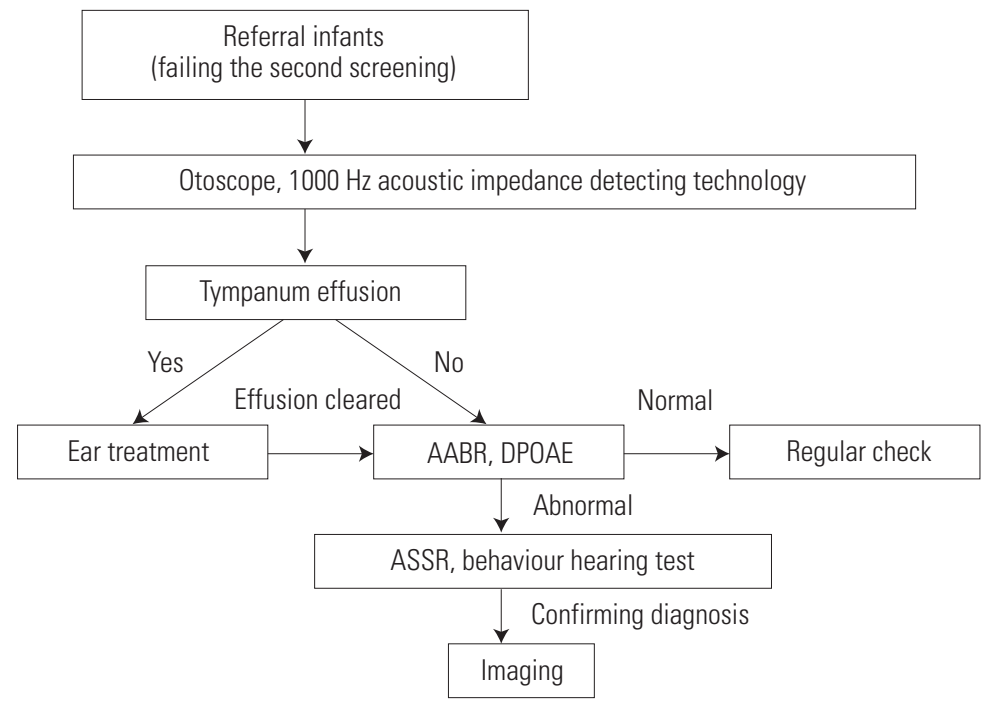

Figure 3. Statistics of universal newborn hearing screening program in Shanghai, 2002-2007

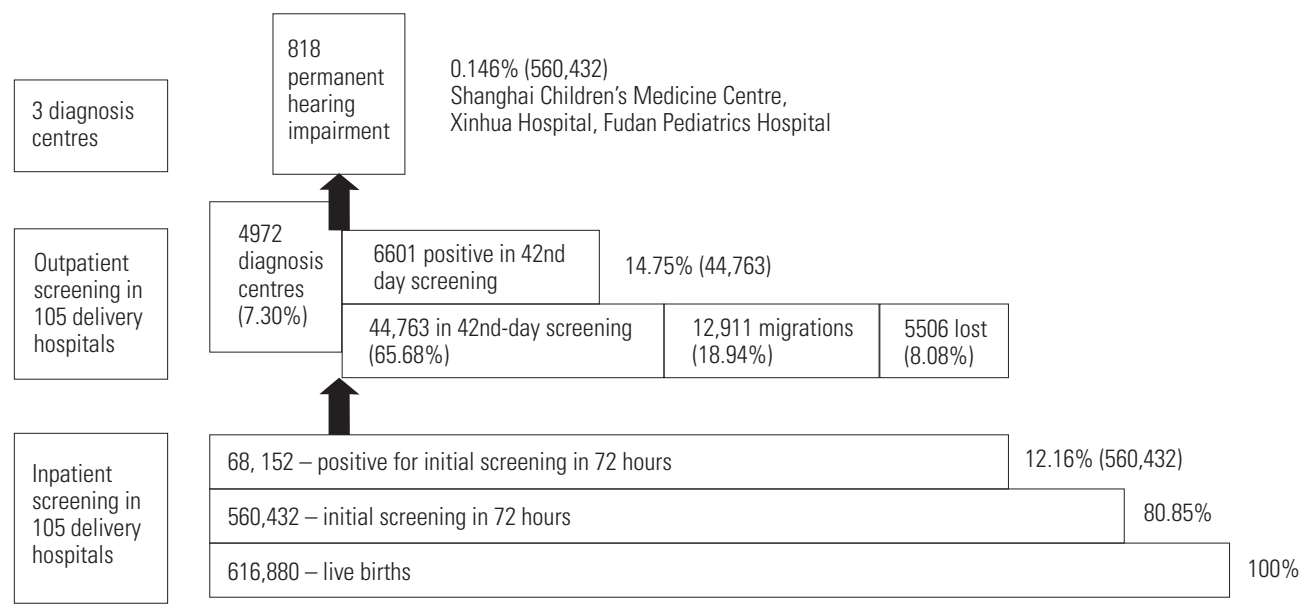

An accounting of the $34.32 \%(23,389)$ of neonates who failed to participate in the second screening despite receiving positive results in the first screening revealed $7.30 \%$ (4972) were taken directly to diagnosis centres after their first screenings, 8.08\% (5506) were lost to contact, and $18.94 \%(12,911)$ were infants of temporary workers who had moved back their original residential provinces following their child's birth (Figure 3).

To help understand the success of our high rate of participation in both stages of screening, it is 
useful to know that annually 51\% of the 100,000 infants born in Shanghai are children of parents who have moved to the city to take temporary work. These parents usually return to their home province after childbirth. If they then return to Shanghai after recuperating from delivery, their newborns are generally left with the grandparents in the home province. Ninety percent of the infants born to these temporary workers, known as the "floating population," are not taken to their birth hospital for a return visit on Day 42.

Among Shanghai residents, failure to return for a second screening on Day 42 (by the $15 \%$ of the remaining $49 \%$ of infants and families who did not follow up) was attributed to economic concern, lack of faith in the process, fear of getting in trouble because of a negative diagnosis, and the like.

Of the infants referred to diagnostic centres, 1.46 per 1000 of those from the first-stage screening (a total of 818) received a diagnosis of permanent hearing impairment within three months of birth. The majority of diagnoses, however, showed slight to moderate hearing loss in referred infants (33.98\%, or 278 infants, and $55.37 \%$ or 453 infants, respectively) with only $3.42 \%$ ( 28 infants) found to have extremely severe hearing loss. Bilateral deafness was diagnosed in $82.40 \%$ of the referred newborns (674) and unilateral deafness in $17.60 \%$ (144) of the infants (Table 2).

Table 1. Number of newborns screened in program, 2002-2007

\begin{tabular}{|c|c|c|c|c|c|c|c|c|}
\hline & $\begin{array}{c}\text { March } \\
\text { - December } \\
2002\end{array}$ & 2003 & 2004 & 2005 & 2006 & $\begin{array}{c}\text { January } \\
\text { - June } \\
2007\end{array}$ & Total \\
\hline \multicolumn{2}{|l|}{ Live births } & 67,418 & 83,817 & 114,072 & 125,130 & 144,994 & 81,449 & 616,880 \\
\hline \multirow[t]{2}{*}{ 3rd day screening } & Number & 56,191 & 78,595 & 105,873 & 117,371 & 129,738 & 72,664 & 560,432 \\
\hline & Positive & 7040 & 8771 & 12,147 & 13,812 & 16,836 & 9546 & 68,152 \\
\hline \multicolumn{2}{|c|}{ 3rd day screening rate $(\%)$} & 83.35 & 93.88 & 92.81 & 93.80 & 89.48 & 89.19 & 90.85 \\
\hline \multicolumn{2}{|c|}{ 3rd day screening positive rate $\%$} & 12.53 & 11.16 & 11.47 & 11.77 & 12.98 & 13.14 & 12.16 \\
\hline \multirow[t]{2}{*}{ 42nd day screening } & Number & 4500 & 5534 & 8264 & 9545 & 10,651 & 6269 & 44,763 \\
\hline & Positive & 468 & 751 & 1359 & 1444 & 1638 & 941 & 6601 \\
\hline \multicolumn{2}{|c|}{ 42nd day screening rate (\%) } & 63.92 & 63.09 & 68.03 & 69.11 & 63.26 & 65.67 & 65.68 \\
\hline \multicolumn{2}{|c|}{$\begin{array}{l}\text { 42nd day screening positive } \\
\text { rate (\%) }\end{array}$} & 10.40 & 13.57 & 16.44 & 15.13 & 15.38 & 15.01 & 14.75 \\
\hline \multicolumn{2}{|c|}{ Diagnosis in 3rd month } & 94 & 118 & 153 & 157 & 189 & 107 & 818 \\
\hline \multicolumn{2}{|c|}{ Diagnosis rate $(\%)$ in $3 r d$ month } & 1.67 & 1.50 & 1.45 & 1.34 & 1.46 & 1.47 & 1.46 \\
\hline \multicolumn{2}{|c|}{ Intervention in 6th month } & 80 & 89 & 141 & 139 & 158 & 99 & 706 \\
\hline \multicolumn{2}{|c|}{ Intervention rate $(\%)$ in 6 th month } & 85.11 & 75.42 & 92.16 & 88.54 & 83.60 & 92.52 & 86.31 \\
\hline
\end{tabular}

Among the infants with permanent hearing impairment receiving interventions, $86.31 \%$ (706) had effective interventions within 6 months (Table 1). All bilateral hearing aids, internal ear moulds and batteries were provided free of charge by Shanghai's Municipal Federation of Disabled People. Cochlear implants were provided free of charge by two Shanghai-based nongovernmental organizations: the Inpatient Health Insurance Foundation for Children and the Charity Foundation.

Interviews with 400 randomly selected parents revealed $90 \%$ were satisfied with the screening process and their knowledge of it. At the same time, 110 parents indicated they had gone through a high degree of mental stress upon learning their child had tested positive for hearing loss. 
Table 2. Degree of hearing loss among newborns in Shanghai, 2002-2007

\begin{tabular}{|c|c|c|c|}
\hline & Criteria & Congenital deafness & Proportion (\%) \\
\hline Degree & \multicolumn{3}{|c|}{ Voice frequency $(500,1000,2000 \mathrm{~Hz}$ Pure-tone threshold average (PTA) } \\
\hline Slight & $26-40 \mathrm{dbHL}$ & 278 & 33.98 \\
\hline Moderate & $41-60 \mathrm{dbHL}$ & 453 & 55.37 \\
\hline Severe & $61-90 \mathrm{dbHL}$ & 59 & 7.21 \\
\hline Extremely severe & $>90 \mathrm{dbHL}$ & 28 & 3.42 \\
\hline Total & & 818 & 100 \\
\hline \multicolumn{4}{|c|}{ Unilateral or bilateral } \\
\hline Unilateral & & 144 & 17.60 \\
\hline Bilateral & & 674 & 82.40 \\
\hline Total & & 818 & 100 \\
\hline
\end{tabular}

$\mathrm{dbHL}=$ decibels hearing level.

\section{Discussion}

Our study shows that a government-sponsored public health universal newborn hearing screening program can be effectively developed and implemented in a large, complex city like Shanghai. This is one of the successful models of a public health UNHSP.

The integrated design of our study, following a pilot implementation to determine key study variables, included an initial soon-after-birth inpatient screening, a second screening on an outpatient basis six weeks later, and coordinated diagnosis and intervention services (including free hearing aids, surgical implantation of cochlear devices and aural and language training). It resulted in high rates of participation for both the initial and the second screenings, reduced false-positive rates at second screening, widespread access to participating centres and prompt and effective interventions that placed no economic burden on the infants' parents.

In addition, we believe our study shows that implementing a well-organized program that adheres to uniform screening, training and intervention standards can achieve high compliance rates and high rates of satisfaction from families involved in the program. Our outcomes, such as high participation at first- and second-stage screenings and high percentages of infants receiving diagnoses and interventions in a timely manner as suggested by international guidelines, exceed those of clinical programs conducted in many developed countries. Furthermore, the success of our program spurred the central government of China in 2005 to add the program to its list of legislated screening programs. Our UNHSP model has since been launched in 27 provinces, municipalities and autonomous regions (of a total of 31 in mainland China).

The study's outcomes are not without shortfalls and limitations, however. Why did $9 \%$ of eligible infants not take part in the initial inpatient screening? Although we had a high rate of participation in the third-day screening, we had no mechanism in place to follow up on groups that failed to show up for the second screening, such as the $26.24 \%$ who were the children of "floating population" workers who temporarily move to Shanghai from other provinces. Subsequent efforts will need to investigate whether these infants received diagnoses and, when necessary, appropriate interventions at their local hospitals. We also need to reconcile the reasons and outcomes of the $8.08 \%$ lost to contact following their initial positive screening, and we need to determine ways to improve the intervention phase so that all infants who receive diagnoses of permanent hearing impairment are 
treated within 6 months. Our study intervened in 706 of the 818 cases diagnosed; 112 who had been diagnosed with permanent hearing impairment were left without proper treatment within the appropriate time period. In addition to addressing these questions, we hope to further analyze data from this study to determine the overall cost-effectiveness of the program.

\section{Contributors}

$\mathrm{X}$. Sun was the main author, principal investigator, project manager and developer of the project's systematic design. X. Shen designed the study methodology and assisted in managing the project. D. Zakus assisted with the analysis and write-up. J. Lv oversaw the sociological assessment and the statistical analysis of the study. Z. Xu managed the diagnostic centres and training program, and $\mathrm{H}$. Wu managed operations in the clinical and rehabilitation centres. W. Hsiao gave instructions on the draft.

\section{Acknowledgements}

The authors wish to thank Zhu Liping, Director of the Shanghai Institute for Women and Children's Health Care; Yu Huijun, Director of the Department of Rehabilitation, Shanghai Municipal Federation of Disabled People; and all hospitals, nongovernmental organizations, colleagues and participants who supported or took part in the study. In addition, they wish to thank William Hsiao, K.T. Li Professor of Economics at the School of Public Health, Harvard University in Boston, Massachusetts, for his advice and guidance, and A.M. Menting for assistance in the preparation of the manuscript.

Source of support: Shanghai Municipal Health Bureau

\section{References}

American Academy of Pediatrics, Joint Committee on Infant Hearing. 1995. "1994 Position Statement." Pediatrics 95: 152-6.

American Academy of Pediatrics. 1999. "Newborn and Infant Hearing Loss: Detection and Intervention." Pediatrics 103: 527-30.

Centers for Disease Control and Prevention. 2003. "Infants Tested for Hearing Loss - United States, 19992001.” Morbidity and Mortality Weekly Report 52: 981-4.

Grandori, F. 1999. "The European Consensus Development Conference on Neonatal Hearing Screening (Milan, May 15-16, 1998).” Archives of Otolaryngology - Head \& Neck Surgery 125: 118.

Green, G.E., D.A. Scott, J.M. McDonald, G.G. Woodworth, V.C. Sheffield and R.J.H. Smith. 1999. "Carrier Rates in the Midwestern United States for GJB2 Mutations Causing Inherited Deafness.” JAMA 281: 22116.

Kanne, T.J., L. Schaefer and J.A. Perkins. 1999. "Potential Pitfalls of Initiating a Newborn Hearing Screening Program.” Archives of Otolaryngology - Head \& Neck Surgery 125: 28-32.

Li, J, Z. Xu and A. Tao. 2004. "Otoacoustic Emissions and Auditory Brainstem Response in High Risk Neonatal Hearing Screening.” Journal of Audiology and Speech Pathology 12: 5-7.

Lin, H.C., M.T. Shu, K. Chang and S.M. Bruna. 2002. "A Universal Newborn Hearing Screening Program in Taiwan." International Journal of Pediatric Otorhinolaryngology 63: 209-18.

Morton, C.C. and W.E. Nance. 2006. "Newborn Hearing Screening - A Silent Revolution.” New England Journal of Medicine 354: 2151-64.

National Institutes of Health. 1993. "Early Identification of Hearing Impairment in Infants and Young Children.” NIH Consensus Statement 11(1): 1-24.

Shen, X. 2001. "The Meaning of Developing Neonatal Hearing Screening in China.” Journal of Clinical Pediatrics 19: 67.

Shen, X. 2002. "Neonatal Hearing Screening." Chinese Journal of Pediatrics 40: 56-8.

US Preventive Services Task Force (USPSTF). 2001. "Newborn Hearing Screening: Recommendations and Rationale." American Family Physician 64: 1995-9. 
US Preventive Services Task Force. 2002. "Newborn Hearing Screening: Recommendations and Rationale." American Journal of Nursing 102: 83-9.

Uus, K. and J. Bamford. 2006. "Effectiveness of Population-Based Newborn Hearing Screening in England: Ages of Interventions and Profile of Cases.” Pediatrics 117: 887-93.

Wessex Universal Neonatal Hearing Screening Trial Group. 1998. "Controlled Trial of Universal Neonatal Screening for Early Identification of Permanent Childhood Hearing Impairment.” Lancet 352: 1957-64.

White, K.R. and T.R. Behrens. 1993. "The Rhode Island Hearing Assessment Project: Implications for Universal Newborn Hearing Screening.” Seminars in Hearing 14: 1-122.

White, K.R. 2003. "The Current Status of EHDI Programs in the United States." Mental Retardation and Developmental Disabilities Research Reviews 9: 79-88.

Wu, S. 2000. “Current Research Status of Children's Hearing Impairment.” Foreign Medical Sciences 27: $254-$ 7.

Xu, Z, J. Li, T. Hu, J. Sun and X. Shen X. 2003. "Sensitivity of Distortion Product Otoacoustic Emissions and Auditory Brain-Stem Response in Neonatal Hearing Screening, a Comparative Study." Chinese Medical Journal 83: 278-80.

Zhao, J.R., H.L. Ge and M. Zhen. 1999. "An epidemiological survey on birth deficient and the disabled morbidities of children under 7 years old in Shanghai." Unpublished manuscript.

Zhao, P. 2001. “Neonatal Hearing Screening.” Foreign Medical Sciences 12: 113-16. 